\title{
ANÁLISE DA POTÊNCIA AERÓBIA DE FUTEBOLISTAS POR MEIO DE TESTE DE CAMPO E TESTE LABORATORIAL
}

ANALYSIS OF AEROBIC POWER OF SOCCER PLAYERS THROUGH FIELD TEST AND LABORATORY TEST

ANÁLISIS DE LA CAPACIDAD AERÓBICA DE FUTBOLISTAS POR MEDIO DE TEST DE CAMPO

Y TESTEN LABORATÓRIO

Cristian Javier Ramirez Lizana (Educador Físico)

Felipe Belozo' (Educador Físico)

Thiago Lourenço ${ }^{3}$ (Educador Físico)

René Brenzikofer ${ }^{2}$ (Físico)

Denise Vaz Macedo ${ }^{3}$ (Bióloga)

Milton ShoitiMisuta ${ }^{4}$

(Educador Físico)

Alcides José Scaglia ${ }^{1}$

(Educador Físico)

1. LEPE, Faculdade de Ciências Aplicadas, UNICAMP, Limeira, SP, Brasil. 2. LIB, Faculdade de Educação Física, UNICAMP, Campinas, SP, Brasil.

3. LABEX, Instituto de Biologia, UNICAMP, Campinas, SP, Brasil. 4. LBI, Faculdade de Ciências Aplicadas, UNICAMP, Limeira, SP, Brasil.

\section{Correspondência:}

Estrada do Morro Azul, 433, Casa $252,13148-302$ - Paulínia, SP, Brasil. crlizana1@gmail.com

\section{RESUMO} protocolo nas avaliações subsequentes.

Palavras-chave: futebol, consumo de oxigênio, fisiologia.

\section{ABSTRACT}

Introdução: Há métodos diretos e indiretos que são utilizados pelos clubes de futebol para avaliar, acompanhar e determinar o $\mathrm{VO}_{2 \max }$ dos jogadores, sendo este muito importante para o rendimento e a recuperação dos atletas durante uma partida. Objetivo: Avaliar o nível de correlação entre as medidas de $\mathrm{VO}_{2 \max }$ por meio da análise direta de gases e do teste de campo Yo-Yo Intermitente Recovery Level 1 (Yo-YoIR1). Métodos: Participaram do estudo 24 jogadores de futebol da categoria SUB-20 de um clube

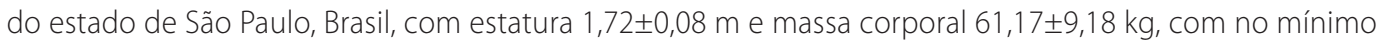
cinco anos de prática na modalidade. Os atletas realizaram o teste de análise direta dos gases em esteira ergométrica e após 48 horas foi realizado o Yo-Yo IR1. Resultados: Os resultados apontaram uma correlação significante entre os testes $(r=0,524 ; p<0,01)$, porém o Yo-Yo IR1 subestimou as medidas de análise direta dos gases em laboratório (44,98ml $/ \mathrm{kg} / \mathrm{min}$ e $48,14 \mathrm{ml} / \mathrm{kg} / \mathrm{min}$, respectivamente). Conclusão: Os resultados apontaram uma correlação moderada entre as medidas de $\mathrm{VO}_{2 \text { max, }}$ dessa forma pode-se utilizar ambos os testes para análise da potência aeróbia dos jogadores de futebol, desde que seja repetido o mesmo

Introduction: There are direct and indirect methods that are used by soccer clubs to assess, monitor and determine the $\mathrm{VO}_{2 \max }$ of soccer players, which is very important for the performance and recovery of athletes during a match. Objective: The aim of this study was to assess the level of the correlation between measures of $\mathrm{VO}_{2 \max }$ by direct gas analysis and by the field test Yo-Yo Intermittent Recovery Level 1 (YO-YO IR1). Methods: The study included 24 Brazilian male soccer players from under 20 category, of a club in the State of São Paulo, Brazil with stature of $1.72 \pm 0.08$ meter and body mass of $61.17 \pm 9.18 \mathrm{~kg}$, with at least five years of practice in the sport. The athletes performed the direct analysis of gases test on a treadmill 48 hours later the YO-YO IRI was performed. Results: The results revealed a significant correlation between tests $(r=0.524 ; p<0.01)$, but Yo-YO IRI underestimated measures of direct analysis of gases in the laboratory $(44.98 \mathrm{~m} / \mathrm{kg} / \mathrm{min}$ and $48.14 \mathrm{ml} / \mathrm{kg} / \mathrm{min}$, respectively). Conclusion: The results showed a moderate correlation between measures of $\mathrm{VO}_{2 \text { max }}$ that way both tests can be used to analyze the aerobic power of soccer players, since the same protocol is repeated in subsequent evaluations.

Keywords: soccer, oxygen consumption, physiology.

\section{RESUMEN}

Introducción: Los métodos directos e indirectos son utilizados por los clubes de fútbol para evaluar, monitoreary determinar el $\mathrm{VO}_{2}$ max de los jugadores, lo que es muy importante para el rendimiento y la recuperación de los atletas durante un partido. Objetivo: Evaluar el nivel de correlación entre las medidas de $\mathrm{VO}_{2 \max }$ de análisis directo de gases y la prueba de campo Yo-Yo Intermitente Recovery Nivel 1 (Yo-YoIR1). Métodos: El estudio incluyó a 24 futbolistas categoría SUB-20 de un club en el Estado de São Paulo, Brasil, con estatura de 1,72 $\pm 0,08$ metro y masa corporal de $61,17 \pm 9,18 \mathrm{Kg}$, con al menos cinco años de práctica en la modalidad. Los atletas realizaron el análisis directo de los gases en una caminadora y después de 48 horas se realizó el Yo-Yo IRT. Resultados: Los resultados revelaron una correlación significativa entre los ensayos ( $r=0,524, p<0,01)$, pero el Yo-Yo IR1 ha subestimado las mediciones de laboratorio del análisis directo de gases (44,98 m//Kg/min. y 48,14 m/ $/ \mathrm{Kg} / \mathrm{min}$., respectivamente). Conclusión: Los resultados indicaron una correlación moderada entre las medidas de $\mathrm{VO}_{2 \max }$ de esta manera se pueden utilizar ambas las pruebas para analizar la capacidad aeróbica de los jugadores de fútbol, siempre que se repita el mismo protocolo en las evaluaciones posteriores.

Palabras Clave: fútbol, consumo de oxígeno, fisiología. 


\section{INTRODUÇÃO}

A constante evolução dos resultados nas diversas modalidades esportivas (cíclicas, acíclicas e coletivas) deve-se em grande parte a produção tecnológica e de conhecimento aplicado ao esporte. O futebol de campo, devido as suas ações de saltar, acelerar e mudar de direção, é considerado uma modalidade muito complexa, classificada como intermitente devido as suas corridas de altas e médias intensidades e com corridas de baixas intensidades para a recuperação passiva $a^{1-7}$.

A potência aeróbia $\left(\mathrm{VO}_{2 \max }\right)$ é considerada muito importante para os jogadores de futebol para que suportem os 90 minutos de jogo, pois ela assegura um alto rendimento energético durante toda a partida e remove rapidamente o lactato acumulado entre as ações com altas intensidades ${ }^{1-4,8}$. O VO ${ }_{2 \max }$ é a máxima capacidade do organismo em captar, transportar e utilizar o oxigênio e pode ser expresso em termos absolutos (l/min) ou relativo à massa corpórea $(\mathrm{ml} / \mathrm{kg} / \mathrm{min})^{2,9}$. Trabalhos de corridas contínuas e intervaladas são utilizados nos treinamentos para melhorar o $\mathrm{VO}_{2 \max }$ dos jogadores, dada à importância da potência aeróbia para os futebolistas; porém atualmente, respeitando a especificidade da modalidade, os treinos com jogos reduzidos estão sendo utilizados para otimizar essa melhora ${ }^{10-14}$.

Com o objetivo de monitorar o nível de desempenho e individualizar os treinamentos, os métodos de avaliação das capacidades físicas tem um papel fundamental. As avaliações físicas foram ao longo do tempo se tornando cada vez mais específicas para cada modalidade, e mais precisa devido à tecnologia disponível. Dentre vários métodos aplicados no futebol, dois são muito utilizados pelos clubes, treinadores e fisiologistas, para a determinação do $\mathrm{VO}_{2 \max }$ dos atletas; um deles é análise direta dos gases por meio do teste incremental de velocidade em esteira ergométrica ${ }^{3,4,9,15}$ e o outro é o teste de campo Yo-Yo Intermittent Recovery Level 1 (Yo-Yo IR1) ${ }^{1,16}$. Os dois testes possuem características diferentes. Embora o teste de análise direta de gases seja considerado o método padrão ouro ${ }^{17}$, não é um teste de fácil aplicação. Deve ser realizado em sala climatizada, com esteira ergométrica especializada, o que impede, por exemplo, a mudança de direção na corrida. Os atletas devem utilizar uma máscara para captação dos gases e devem calçar tênis. Já o Yo-Yo IR1 exige apenas um aparelho de som para reproduzir o áudio do CD e uma trena para medir a distância entre os cones. O teste permite ações motoras próximas do jogo de futebol e pode ser realizado no campo com os atletas calçando chuteiras.

Sabe-se que no treinamento esportivo é muito importante respeitar o principio da especificidade, em que o treino e as avaliações físicas devem ser realizados sobre os requisitos específicos do desempenho desportivo, em termos de ambiente, segmento corporal e coordenações psicomotoras.

Através de protocolos incrementais em esteiras é possível verificar a intensidade na qual os sujeitos atingem o máximo de sua capacidade de captar, transportar e metabolizar o oxigênio $\left(\mathrm{VO}_{2 \text { max }}\right)$, e devido a este fato o método proposto é considerado o mais adequado para mensurar esta capacidade. Porém, devido às limitações impostas pela realidade da grande maioria dos clubes, as análises diretas se tornam de difícil utilização. Por isso, pesquisadores propuseram protocolos indiretos para a mensuração do $\mathrm{VO}_{2 \max }$ entre eles o Yo-Yo IR1, e dada a especificidade e a praticidade na execução, este protocolo é atualmente amplamente utilizado ${ }^{4}$.

Na literatura não há um consenso sobre o protocolo de análise direta dos gases mais adequado para mensurar a potência aeróbia de futebolistas. Em alguns casos os protocolos utilizados foram validados utilizando sujeitos de outras modalidades ${ }^{18}$. Portanto, o objetivo do estudo é avaliar a possível correlação entre as medidas de $\mathrm{VO}_{2 \max }$ através da análise direta de gases em protocolo validado com corredores ${ }^{9} \mathrm{e}$ do teste de campo Yo-Yo IR1'.

\section{MÉTODOS}

O presente estudo foi realizado com 24 futebolistas da categoria SUB20 de um clube federado no estado de São Paulo, Brasil, com estatura média 1,72 \pm 0,08 m e massa corporal 61,17 $\pm 9,18$ kg, com no mínimo com cinco anos de prática na modalidade. Os atletas realizaram o protocolo incremental de análise direta de gases ${ }^{9}$ em esteira ergométrica (Inbrasport, modelo ATL 2000, Porto Alegre, RS, Brasil), com velocidade inicial de $9 \mathrm{~km} \cdot \mathrm{h}^{-1}$, e inclinação de $1 \%$. O incremento foi de $0,3 \mathrm{~km} \cdot \mathrm{h}^{-1}$ a cada 25 segundos. $O$ teste foi realizado até a exaustão voluntária. Durante a avaliação foram monitoradas continuamente as variáveis: frequência cardíaca (marca Polar, modelo RS100, Copenhagen, Dinamarca), consumo de oxigênio $\left(\mathrm{VO}_{2}\right)$, produção de gás carbônico $\left(\mathrm{VCO}_{2}\right)$ e taxa de troca respiratória (RER). Os parâmetros cardiorrespiratórios $\left(\mathrm{VO}_{2}, \mathrm{VCO}_{2}\right.$ e RER) foram mensurados na respiração através do analisador de gases (CPX/D MedGráfics, St. Paul, Minnesota, EUA) que foi calibrado automaticamente conforme especificações do fabricante. O teste foi realizado em laboratório com temperatura e umidade controladas. Para o método indireto foi selecionado o teste Yo-Yo IR1 ${ }^{1}$, realizado em um campo de futebol, com os atletas calçando chuteiras. Cones foram utilizados para demarcar o espaço para corrida, bem como uma fita métrica para determinar os 20 metros de distância da corrida e os cinco metros de recuo para a recuperação. Os atletas deveriam percorrer a distância de $20 \mathrm{~m}$, ida e volta (40 m), dentro do tempo estipulado por um sinal sonoro (figura 1). O intervalo de tempo foi diminuindo ao longo do teste, aumentando a intensidade da corrida. Os participantes tiveram $10 \mathrm{~s}$ de intervalo entre cada esforço, e neste tempo deveriam trotar até o cone do recuo e se posicionar para uma nova saída. O teste foi interrompido quando os atletas não conseguiram mais suportar a intensidade de corrida. Um aparelho de som foi utilizado para reproduzir o áudio do CD específico para o teste. Para a execução de ambos os testes foi respeitado um período mínimo de repouso de 48 horas. Os dados foram tratados em ambiente Matlab. Por meio do teste de lilliefors verificamos que os dados não apresentaram uma distribuição normal, dessa forma a análise de correlação foi realizada por meio do teste de Spearman e para verificar a diferença entre as médias foi realizado um teste de hipótese por meio do método de Wilcoxon.

A Pesquisa foi aprovada pelo Comitê de Ética em Pesquisa da Faculdade de Ciências Médicas da Unicamp em 19/07/2012, sob parecer de número 62368/2012 e CAAE: 03276612.2.0000.5404.

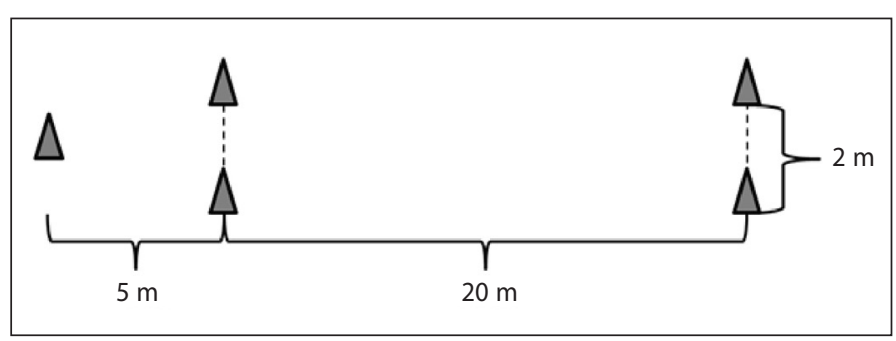

Figura 1. Esquema do teste Yoyo IR1.

\section{RESULTADOS}

Após a avaliação dos 24 atletas realizando o método indireto (Yo-Yo IR1) e o método direto (análise direta dos gases em laboratório) para determinação da potência aeróbia, encontrou-se uma média de $\mathrm{VO}_{2 \max }$ de $44,98 \mathrm{ml} / \mathrm{kg} / \mathrm{min}$ para o teste indireto e $48,14 \mathrm{ml} / \mathrm{kg} / \mathrm{min}$ no teste direto (tabela 1). Nos gráficos de histograma observa-se que as medidas do teste indireto não possuem uma distribuição normal, (figura 2), ao contrário das medidas do teste direto (figura 3). Após o tratamento dos dados encontrou-se também um coeficiente de correlação de Spearman de 0,524 com um valor de significância de $p<0,01$, entre os valores de 
$\mathrm{VO}_{2 \max }$ obtidos nos diferentes testes (figura 4). Na tabela 1 encontram-se os valores das médias e do intervalo de confiança para os testes direto e indireto. Os resultados mostram por meio do teste de Wilcoxon que o teste indireto subestima as medidas do teste direto $(44,98 \mathrm{ml} / \mathrm{kg} / \mathrm{min}$ e $48,14 \mathrm{ml} / \mathrm{kg} / \mathrm{min}$, respectivamente, $\mathrm{p}<0,001$ ) (figura 5 ).

Tabela 1. Médias e intervalos de confiança dos métodos indireto e direto ( $\mathrm{ml} / \mathrm{kg} / \mathrm{min}$ ).

\begin{tabular}{c|c|c|c}
\hline & Média & \multicolumn{2}{|c}{ Intervalo Confiança } \\
\hline Indireto & 44,98 & 44,09 & 45,87 \\
\hline Direto & 48,14 & 46,34 & 49,95 \\
\hline
\end{tabular}

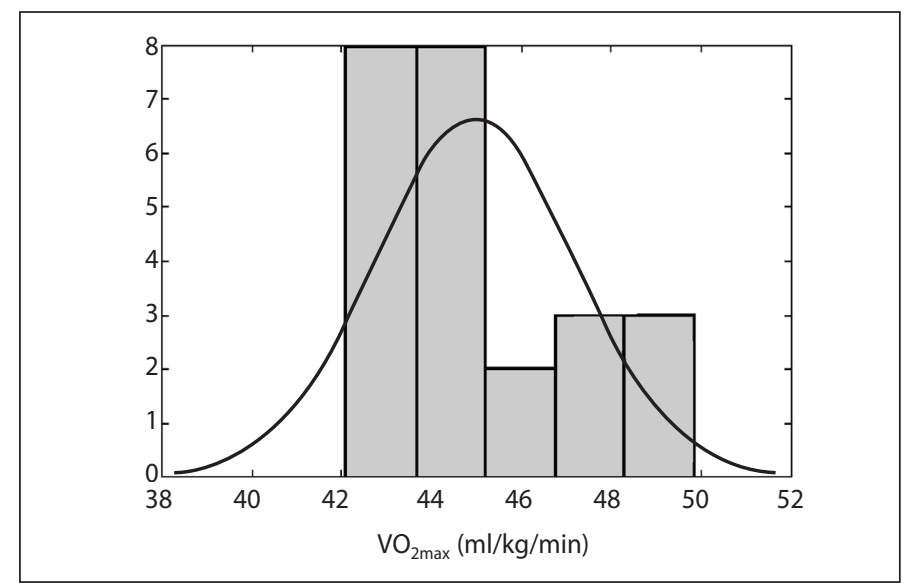

Figura 2. Histograma das medidas de $\mathrm{VO}_{2 \max }$ indireto, a amostra não possui uma distribuição normal.

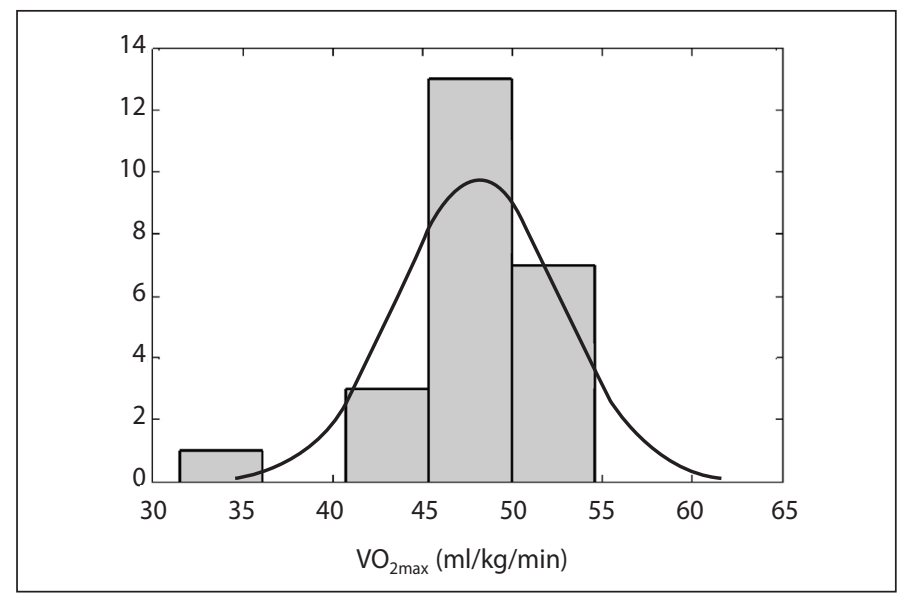

Figura 3. Histograma das medidas de $\mathrm{VO}_{2 \max }$ direto, a amostra possui uma distribuição normal.

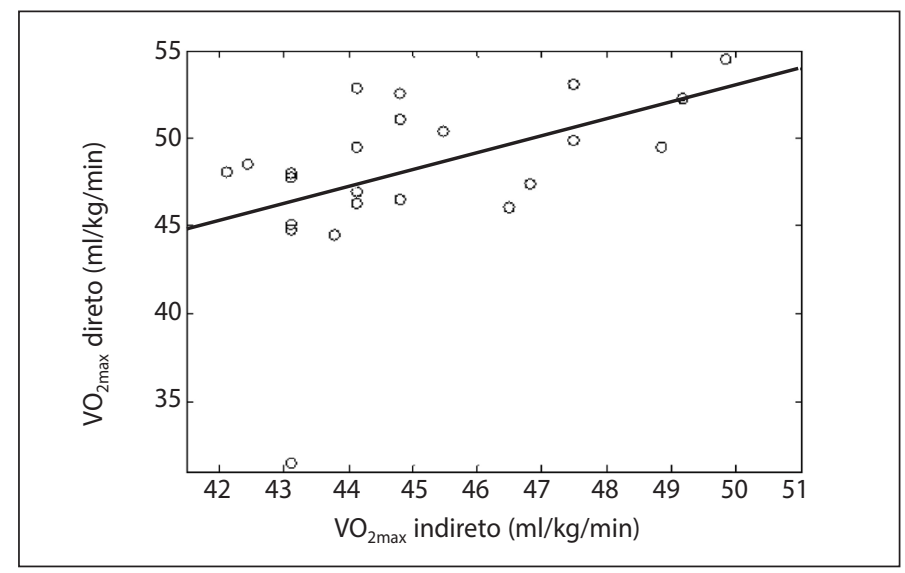

Figura 4. Distribuição das medidas diretas e indiretas. Coeficiente de Spearman $0,542(p<0,01)$.

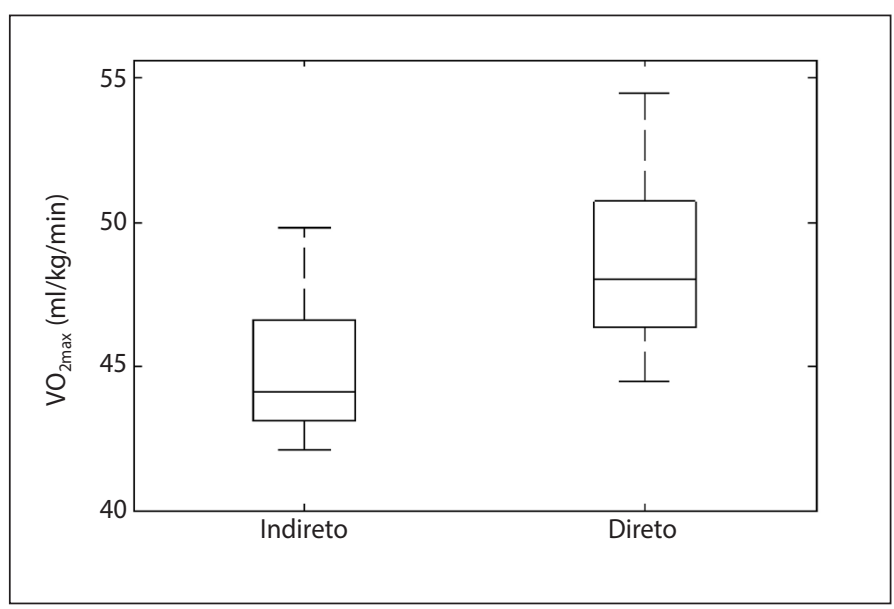

Figura 5. Boxplot das medidas indiretas e diretas de $\mathrm{VO}_{2 \max }$. Diferença significante com $p<0,001$.

\section{DISCUSSÃO}

Após a análise dos dados da potência aeróbia em atletas de futebol, através do método direto e indireto, percebe-se uma diferença de valores obtidos no $\mathrm{VO}_{2 \text { max }}$ em que o teste indireto subestima as medidas do teste direto. As diferenças entre os valores encontrados nos testes também foram encontradas nos estudos de Metaxas et al ${ }^{16}$. Nesse estudo os autores avaliaram a potência aeróbia $\left(\mathrm{VO}_{2 \max }\right)$ em atletas de futebol entre os testes de campo (Yo-Yo test endurance (T1) e o Yo-Yo Test intermitente com ergoespirometria portátil (T2)) e os testes laboratoriais em esteira ergométrica (contínuo (T3) e intermitente (T4)). $\mathrm{O}$ autor observou que os valores de $\mathrm{VO}_{2 \max }$ realizados sem ergoespirometria (T1) foram mais baixos em relação aos demais (T2,T3,T4). Tal fato justifica-se pela precisão dos analisadores de gases nos valores de $\mathrm{VO}_{2 \text { max }}$, bem como aos diferentes ambientes em que os testes foram realizados. Os estudos de Cetolin et al ${ }^{19}$ apontam que o mesmo teste aplicado em terrenos diferentes provoca diferentes solicitações metabólicas e exigências fisiológicas, acarretando assim em alterações nos resultados finais. Em seus trabalhos com o teste de TCAR realizado na grama e em solo arenoso, os autores perceberam que o pico de velocidade no solo arenoso é menor do que na grama, enquanto que a concentração de lactato [La] é maior na areia devido ao maior esforço físico para se deslocar em solos com menor resistência.

Embora os valores de $\mathrm{VO}_{2 \max }$ sejam menores nos teste realizados em campo, Metaxas et all ${ }^{16}$ afirmaram que o Yo-Yo test deve ser utilizado pelos treinadores porque são ferramentas fáceis e baratas e que respeitam a especificidade da modalidade, pois além do teste exigir as ações que acontecem no jogo (corrida intermitente e mudança de direção), a avaliação é realizada no ambiente de jogo com chuteiras, vestimentas e temperatura próximas à realidade da modalidade ${ }^{20,21}$. Em contrapartida, apesar do teste de análise direta dos gases ${ }^{9}$ ter sido validado com corredores de rua e realizado em ambiente laboratorial, o mesmo devido a acurácia nos valores pode ser considerado o mais preciso para a avaliação do $\mathrm{VO}_{2 \text { max }}$ pois o protocolo adotado utiliza medidas diretas de consumo de $\mathrm{O}_{2}$ e produção de $\mathrm{CO}_{2}$ para mensurar o $\mathrm{VO}_{2 \text { max }}$. Por meio do teste proposto por Lourenço ${ }^{9}$ é possível prescrever e controlar os treinos em diferentes intensidades, pois com as velocidades de limiar ventilatório e $\mathrm{VO}_{2 \max }$ é possível estabelecer zonas de treinamento (baixa intensidade, intensidade moderada e alta intensidade) 22.

Esses fatos nos levam a considerar que o teste mais adequado para avaliar $0 \mathrm{VO}_{2 \max }$ estará diretamente relacionado aos objetivos e a condição financeira da equipe, pois a grande parte dos testes em laboratório demandam uma grande quantidade de dias para coletas e também possuem um alto valor financeiro. Isso também pode ser 
verificado nas avaliações para determinação do limiar anaeróbio (Lan), onde a máxima fase estável de lactato (MFEL) ${ }^{23}$ é considerada como o "padrão ouro" de avalição, mas devido ao elevado número de dias para a coleta, outros testes como o TCAR e o teste de lactato mínimo, são mais utilizados para a determinação do Lan²4,25.

No caso do futebol, em que as avaliações físicas são realizadas varias vezes durante a temporada para prescrição, avaliação e reavaliação dos treinamentos ${ }^{22}$, os clubes necessitam de metodologias eficazes, simples, de baixo custo e que sejam específicas para o futebol. São poucos os clubes que possuem uma esteira, uma sala e equipamentos para análise direta de gases, porém todos os clubes facilmente podem obter o áudio do Yo-Yo IR1 e uma fita métrica para aplicar o teste.

Apesar das dificuldades na busca em encontrar o teste mais adequado para avaliar a potência aeróbia em futebolistas, nossos dados apresentam uma correlação significante entre os métodos, o que permite a utilização do Yo-Yo IR1 para análise da potência aeróbia. Além disso, os estudos de Carminatti et a ${ }^{23}$ e Bangsbo et al ${ }^{1}$ reforçam a importância da utilização do Yo-Yo Test para a determinação da potência aeróbia, por se tratar de um método de baixo custo com alta especificidade para com a modalidade do futebol.

\section{CONCLUSÃO}

Verificando os dados do teste de $\mathrm{VO}_{2 \text { max }}$ tanto direto quanto indireto, percebemos que ambos têm vantagens e desvantagens, e a diferença de valores entre os métodos acontece devido as mudanças de ambiente, materiais e características da modalidade. Portanto é importante que as equipes de futebol escolham avaliações físicas fidedignas e que respeitem o princípio da especificidade para com a modalidade. Assim, o método escolhido deve buscar uma semelhança com as ações realizadas no jogo (mudanças de direção, saltos, corridas intermitentes e temperaturas ambientes).

Todos os autores declararam não haver qualquer potencial conflito de interesses referente a este artigo.

\section{REFERÊNCIAS}

1. Bangsbo J, laia FM, Krustrup P. The Yo-Yo intermittent recovery test: a useful tool for evaluation of physical performance in intermittent sports. Sports Med. 2008;38(1):37-51. Epub 2007/12/18.

2. Hoff J, Wisloff U, Engen LC, Kemi OJ, Helgerud J. Soccer specific aerobic endurance training. British journal of sports medicine. 2002;36(3):218-21.

3. McMillan K, Helgerud J, Macdonald R, Hoff J. Physiological adaptations to soccer specific endurance training in professional youth soccer players. British journal of sports medicine. 2005;39(5):273-7.

4. Stolen T, Chamari K, Castagna C, Wisloff U. Physiology of soccer: an update. Sports Med. 2005;35(6):501-36.

5. Juel C, Klarskov C, Nielsen JJ, Krustrup P, Mohr M, Bangsbo J. Effect of high-intensity intermittent training on lactate and $\mathrm{H}+$ release from human skeletal muscle. American journal of physiology Endocrinology and metabolism. 2004;286(2):E245-51.

6. Bangsbo J. The physiology of soccer--with special reference to intense intermittent exercise. Acta physiologica Scandinavica Supplementum. 1994;619:1-155.

7. Silva JF, Guglielmo LGA, Floriano LT, Arins FB, Dittrich N. Aptidão aeróbia e capacidade de sprints repetidos no futebol: comparação entre as posições. Motriz. 2009;15(4):861-70.

8. Ziogas GG, Patras KN, Stergiou N, Georgoulis AD. Velocity at lactate threshold and running economy must also be considered along with maximal oxygen uptake when testing elite soccer players during preseason. Journal of strength and conditioning research / National Strength \& Conditioning Association. 2011;25(2):414-9.

9. Lourenco TF, Martins LE, Tessutti LS, Brenzikofer R, Macedo DV. Reproducibility of an incremental treadmill VO(2)max test with gas exchange analysis for runners. Journal of strength and conditioning research / National Strength \& Conditioning Association. 2011;25(7):1994-9.

10. Hill-Haas SV, Dawson B, Impellizzeri FM, Coutts AJ. Physiology of small-sided games training in football: a systematic review. Sports Med. 2011;41(3):199-220.

11. Hill-Haas SV, Dawson BT, Coutts AJ, Rowsell GJ. Physiological responses and time-motion characteristics of various small-sided soccer games in youth players. Journal of sports sciences. 2009;27(1):1-8.

12. Impellizzeri FM, Marcora SM, Castagna C, Reilly T, Sassi A, laia FM, et al. Physiological and performance effects of generic versus specific aerobic training in soccer players. International journal of sports medicine. 2006;27(6):483-92.
13. Hill-Haas SV, Coutts AJ, Rowsell GJ, Dawson BT. Generic versus small-sided game training in soccer. International journal of sports medicine. 2009;30(9):636-42.

14. Pasquarelli BN, Souza VAFA, Stanganelli LCR. Os jogos com campo reduzido no Futebol. Rev Bras Futebol. 2010;3:02-27

15. Dupont G, Akakpo K, Berthoin S. The effect of in-season, high-intensity interval training in socce players. Journal of strength and conditioning research / National Strength \& Conditioning Association. 2004;18(3):584-9. Epub 2004/08/24.

16. Metaxas TI, Koutlianos NA, Kouidi EJ, Deligiannis AP. Comparative study of field and laboratory tests for the evaluation of aerobic capacity in soccer players. Journal of strength and conditioning research / National Strength \& Conditioning Association. 2005;19(1):79-84. Epub 2005/02/15.

17. Costa EC. Validade da medida do consumo máximo de oxigênio predito pelo teste de cooper de 12 minutos em adultos jovens sedentários. Motricidade. 2008;4(3):5-10.

18. da Silva JF, Guglielmo LGA, Dittrich N, Floriano LT, Arins FB. Relação entre aptidão aeróbia e capacidade de sprints repetidos no futebol: efeito do protocolo. Rev Bras Cineantropom Desempenho Hum. 2011;13(2):111-6.

19. Cetolin T, Foza V, Carminatti L, Guglielmo LGA, Fernades da Silva J. Diferença entre intensidade do exercício prescrita por meio do teste TCAR no solo arenoso e na grama. Rev Bras Cineantropom Desempenho Hum. 2010;12:29-35.

20. Zagatto AM, Miyagi WE, Sakugawa RL, Papoti M. Utilização da distância total percorrida no teste específico de hoff como preditor da velocidade de limiar anaeróbio no futebol. Revista Brasileira de Medicina do Esporte. 2013;19:267-70.

21. Chamari K, Hachana Y, Kaouech F, Jeddi R, Moussa-Chamari I, Wisløff U. Endurance training and testing with the ball in young elite soccer players. Br J Sports Med. 2005;39(1):24-8.

22. Mendez-Villanueva A, Buchheit M. Football-specific fitness testing: adding value or confirming the evidence? Journal of sports sciences. 2013;31(13):1503-8.

23. Carminatti LJ, Possamai CA, de Moraes M, da Silva JF, de Lucas RD, Dittrich N, et al. Intermittent versus Continuous Incremental Field Tests: Are Maximal Variables Interchangeable? Journal of sports science \& medicine. 2013;12(1):165-70. Epub 2013/10/24. 